\title{
Effect of Varieties, Cutting and Nitrogen Management on Green Fodder Yield, Nutrient Uptake, Available Soil Nutrient Status and Economics of Dual Purpose Pearl Millet (Pennisetum glaucum L.)
}

\author{
Manjanagouda S. Sannagoudar*, B.S. Lalitha, G.K. Prajwal Kumar, \\ Prashant and V. Bhavya
}

Department of Agronomy, College of Agriculture, UAS, GKVK, Bangalore- 560065, Karnataka, India

*Corresponding author

\author{
A B S T R A C T
}

A field experiment was conducted during kharif2014 at Zonal Agricultural Research Station, VCFarm, Mandya to study the effect of varieties, cutting and nitrogen management on yield, nutrient uptake, available soil nutrient status and economics of dual purpose pearl millet (Pennisetum glaucum L.). The soil of the experimental site was red sandy loam with medium available nitrogen, phosphorus and potassium level. Experiment was laid out in randomized complete block design with factorial concept replicated thrice.

\section{Keywords}

Varieties, Cutting,

Nitrogen, Green

fodder, Nutrient uptake, Economics.

Article Info

Accepted:

04 October 2017

Available Online:

10 December 2017
There were 12 treatment combinations involving 3 varieties (BAIF Bajra-1, AVKB-19 and GFB-1), two cutting management practices (Single cut at 45 DAS for green fodder and later for grain purpose and two cuts for green fodder at 45 DAS and at 40 days after first cut and later for grain purpose) and two nitrogen levels (100 and $\left.150 \mathrm{~kg} \mathrm{ha}^{-1}\right)$. The results revealed that, BAIF Bajra-1 recorded significantly higher green fodder (365.21 q ha $\left.\mathrm{q}^{-1}\right)$, dry matter yield (92.47 $\mathrm{q} \mathrm{ha}^{-1}$ ), higher nitrogen, phosphorus and potassium uptake (174.26, $17.85,142.75 \mathrm{~kg} \mathrm{ha}^{-1}$ ) and lower available soil nutrient status (NPK) after harvest (167.57, $\left.22.75,114.21 \mathrm{~kg} \mathrm{ha}^{-1}\right)$. Two cuts for green fodder recorded significantly higher green fodder $\left(334.31 \mathrm{q} \mathrm{ha}^{-1}\right)$, dry matter yield $\left(76.33 \mathrm{q} \mathrm{ha}^{-1}\right)$, but single cut for green fodder later for grain purpose has recorded significantly higher nitrogen, phosphorus and potassium uptake $\left(138.83,14.45,117.32 \mathrm{~kg} \mathrm{ha}^{-1}\right)$ and lower available soil nutrient status after harvest $\left(163.81,21.85,112.17 \mathrm{~kg} \mathrm{ha}^{-1}\right)$ respectively. Nitrogen at $150 \mathrm{~kg} \mathrm{ha}^{-1}$ given significantly higher green fodder $\left(316.91 \mathrm{q} \mathrm{ha}^{-1}\right)$, dry matter yield $\left(74.95 \mathrm{q} \mathrm{ha}^{-1}\right)$ and higher nitrogen, phosphorus and potassium uptake (146.33, 15.51, $\left.126.54 \mathrm{~kg} \mathrm{ha}^{-1}\right)$ and higher available soil nutrient status after harvest of the crop $\left(170.55,23.09,116.15 \mathrm{~kg} \mathrm{ha}^{-1}\right)$. Higher gross returns ( $\left.\square 60102 \mathrm{ha}^{-1}\right)$, net returns $\left(\square 42928 \mathrm{ha}^{-1}\right.$ ) and B: C ratio (3.49) were recorded in BAIF Bajra-1 with single cut for green fodder followed by harvest for grain purpose along with application nitrogen $150 \mathrm{~kg} \mathrm{ha}^{-1}$.

\section{Introduction}

Animal husbandry is an important component of farming in India. Farmers engage in rearing of animals, often as a subsidiary activity, for the supply of milk, meat, wool and manure, or for using them as work animals. India supports nearly $20 \%$ of the world's livestock and $16.8 \%$ human population with only $2.3 \%$ of the world's geographical area. India is the 
leader in cattle (16\%) and buffalo (5.5\%). The livestock sector contributes $32 \%$ of the agricultural output, which is $22 \%$ of the total GDP in India. Deficiency in feed and fodder has been identified as one of the major components in achieving the desired level of livestock production. The shortage in dry fodder is $21.8 \%$ compared with requirement of 560 million tons for the current livestock population (Anonymous, 2006). Although, India stands first in milk production $(90 \mathrm{mt})$ in the world, but average milk yield is very low (5 litres/animal) compared to developed countries (24 litres/animal). Deficit supply of green fodder is one of the main reasons for low milk yield along with other factors like imbalanced nutrition, good quality fodder (Anonymous, 2009). In India, due to increased population pressure and competition from the food crops for natural resources like land, water, sunlight etc., therefore it is not possible to increase the area under fodder crops further. The only way to bridge the large gap between demand and supply of fodder is through maximizing the fodder production per unit area and unit time and strategies to develop and adopt dual type grain-cum-fodder crop varieties to cater the demand of grain and fodder with available land resource. At this juncture, adopting dual type grain cum fodder varieties gaining importance to overcome green fodder shortage. Pearl millet (Pennisetum glaucum $L$.) is one of the important minor millets is being cultivated for high dietary fibre and nutrient source for human beings and also a good fodder crop for livestock. The dual purpose nature of pearl millet has been recently identified due to its profuse tillering, withstanding capacity for repeated harvesting, absence of anti-nutritional factor like prussic acid, better performance under marginal and low fertile soils (Reddy et al., 2012). Pearl millet, popularly known as poor man's crop due to its fair productivity even under lower management and multicut nature ensures the fodder supply year around and reduced cost of cultivation due to repeated cultivation like in single cut crops. In any crop, selection of good variety will increase the yield to tune of $15-24 \%$. Nitrogen plays an important role in increasing all the growth and growth attributing characters which finally led to increased green fodder yield. In addition nitrogen increases the crude protein content in green fodder. In this regard, scientific study on cutting and nitrogen management on growth and green fodder yield of pearl millet is very meagre. Therefore, the present investigation was under taken on performance of dual purpose pearl millet (Pennisetum glaucum L.) varieties as influenced by cutting and nitrogen management.

\section{Materials and Methods}

Field experiment was conducted during kharif season of 2014 at Zonal Agricultural Research Station, Vishweswaraiah Canal Farm, Mandya (Karnataka) to assess the green fodder yield and quality of dual purpose pearl millet varieties as influenced by cutting and nitrogen management. The experiment was laid out in RCBD with factorial concept replicated thrice. The experiment consisted of 12 treatment combinations viz., three varieties (BAIF Bajra-1, AVKB-19 and GFB-1), two cutting management practices $\left(\mathrm{C}_{1}\right.$-Single cut at 45 days after sowing for green fodder followed by harvest for grain purpose. $\mathrm{C}_{2^{-}}$ Two cuts ( $1^{\text {st }}$ at 45 days after sowing and $2^{\text {nd }}$ at40 days after $1^{\text {st }}$ cut) for green fodder followed by harvest for grain purpose and two levels of nitrogen (100 and $150 \mathrm{~kg} \mathrm{~N} / \mathrm{ha}$ ). Equal quantity of farm yard manure at the rate of $10 \mathrm{t} \mathrm{ha}^{-1}$ was applied to each plot and mixed well in soil three weeks prior to sowing. Furrows were opened at $30 \mathrm{~cm}$ apart, $60 \mathrm{~kg} \mathrm{P}_{2} \mathrm{O}_{5} \mathrm{ha}^{-1}$ and $40 \mathrm{~kg} \mathrm{~K}_{2} \mathrm{O} \mathrm{ha}{ }^{-1}$ were applied through single super phosphate and muriate of potash respectively. Nitrogen as basal $50 \%$ and remaining as top dress applied 
in two equal splits at 45 DAS and 85 DAS in the form of Urea. The crop was sown during $1^{\text {st }}$ week of August and harvested when crop attained $50 \%$ flowering. Five plants were randomly selected in each net plot area for taking observations on growth and yield attributing parameters. The crop in each net plot was harvested separately as per treatment and the values were converted into hectare basis and expressed in quintals. The samples were first dried under shade and then in electric oven at a temperature of $60^{\circ} \mathrm{C}$ till attaining constant weight on the basis of weight of these samples, the green fodder yield was converted into dry matter yield (q/ha).

\section{Nutrient uptake by crop}

\section{Digestion of plant samples}

One gram plant samples were pre-digested with $5 \mathrm{ml}$ nitric acid and digested with di-acid mixture of nitric acid and perchloric acid (9:4). The clean digested material was made up to $50 \mathrm{ml}$ volume with $6 \mathrm{~N} \mathrm{HCl}$ and was used for the analysis of all mineral elements.

\section{Nitrogen uptake}

Nitrogen content was estimated by modified micro-kjeldhal's method as outlined by Jackson (1967) and expressed in percentage. Nitrogen uptake (kg/ha) by crop was calculated for each treatment separately using the following formula.

Nitrogen uptake $\left(\mathrm{kg} \mathrm{ha}{ }^{-1}\right)=$ (Nitrogen concentration (\%) / 100) X Dry matter (kg $\mathrm{ha}^{-1}$ )

\section{Phosphorus uptake}

Phosphorus content in the digested plant sample was estimated by vanadomolybdo phosphoric yellow colour method in nitric acid medium and the colour intensity was measured at $460 \mathrm{~nm}$ wave length as outlined by Jackson (1973). It is calculated using the following formula.

Phosphorus uptake (kg ha-1) = (Phosphorus concentration (\%) / 100) X Dry matter (kg $\left.\mathrm{ha}^{-1}\right)$.

\section{Potassium uptake}

Potassium in the plant samples digest were estimated by atomizing the diluted acid extract in a flame photometer as described by Jackson (1973). It is calculated using the following formula.

Potassium uptake $\left(\mathrm{kg} \mathrm{ha}{ }^{-1}\right)=($ Potassium concentration (\%) / 100) X Dry matter (kg $\mathrm{ha}^{-1}$ ).

\section{Chemical analysis of soil}

Representative soil samples from the experimental plots were drawn from the top $45 \mathrm{~cm}$ depth before sowing of the crop. Similarly, the surface soil samples from 0 to $45 \mathrm{~cm}$ depth were also collected from each experimental plot after harvest of crop. Soil samples collected were air dried in shade, powdered with wooden mallet and passed through $2 \mathrm{~mm}$ sieve and chemically analyzed for organic carbon content $(\%), \mathrm{pH}, \mathrm{EC}\left(\mathrm{dsm}^{-}\right.$ ${ }^{1}$ ), available nitrogen, phosphorus and potassium content of the soil.

\section{Available nitrogen $\left(\mathrm{kg} \mathrm{ha}^{-1}\right)$}

Available nitrogen was determined by alkaline permanganate method as outlined by Subbiah and Asija (1956).

\section{Available phosphorus (kg ha $\left.{ }^{-1}\right)$}

Available phosphorus was determined by Olsen's method as outlined by Jackson (1967). 
Available potassium (kg ha $\left.{ }^{-1}\right)$

Available potassium was determined by Neutral normal ammonium acetate solution using flame photometer as outlined by Jackson (1967).

\section{Economics}

To work out the economic information, market price of different fertilizer and labour units required for establishment of crops and harvesting was considered. Cost of labour was calculated by taking into account the prevailing labour wages at the time of investigation. Gross returns, net returns and benefit cost ratio were worked out by using the following formulae.

Gross returns $=$ green fodder yield $\mathrm{x}$ market price

Net returns $=$ Gross returns - Total cost of cultivation

Gross returns $\left(\mathrm{ha}^{-1}\right)$
Benefit: Cost ratio $=$
Total cost of cultivation $\left(\mathrm{ha}^{-1}\right)$

\section{Statistical analysis of data}

The experimental data collected on growth and yield components of plant was subjected to Fisher's method of "Analysis of Variance" (ANOVA) as outlined by Panse and Sukhatme (1967). Wherever, F- test was significant, for comparison among the treatment means, an appropriate value of critical difference (C.D.) was worked out. If F-test found non-significant, against C.D. values NS (Non-Significant) was indicated. All the data were analyzed and the results were presented and discussed at a probability level of five per cent. Correlation matrix was worked out between growth, yield and quality parameters (Gomez and Gomez, 1984).

\section{Results and Discussion}

\section{Green fodder and dry matter yield ( $\left(\mathrm{ha}^{-1}\right)$}

Among the different varieties the BAIF Bajra1 variety recorded significantly higher green fodder yield and dry matter yield (365.21 \& $92.47 \mathrm{q} \mathrm{ha}^{-1}$ ) over other varieties GFB-1 (295.21 \& $\left.64.90 \mathrm{q} \mathrm{ha}^{-1}\right)$ and AVKB-19 (249.78 \& $43.0 \mathrm{q} \mathrm{ha}^{-1}$ ) respectively (Table 1). The increase in green forage yield in the variety BAIF Bajra-1 was mainly due to significantly higher plant height, number of tillers $\mathrm{m}^{-1}$ row length and leaf: stem ratio. Variety BAIF Bajra-1 accumulated significantly higher dry matter which might be due to increased higher plant height, number of tillers and leaf: stem ratio finally resulted in significantly higher green forage yield. The genetic potential of the variety could helped to excel better when compared to other varieties tried. The results are in conformity with the findings of Bali et al., (1998) and Rana et al., (2009). Significantly lower green forage and dry matter yield with variety AVKB-19 and GFB-1 was due to lower plant height and number of tillers $\mathrm{m}^{-1}$ row growth due to genetic makeup of the varieties.

Two cuts for green fodder followed by harvest for grain purpose has recorded significantly higher green fodder $(334.31 \mathrm{q}$ $\mathrm{ha}^{-1}$ ) and dry matter yield (76.33 q ha ${ }^{-1}$ ) compared to the single cut for green fodder followed by harvest for grain purpose (272.49 $\mathrm{q} \mathrm{ha}^{-1}$ and $57.26 \mathrm{q} \mathrm{ha}^{-1}$ respectively) (Table 1). It was due to two cut for green fodder followed by harvest for grain purpose facilitates the more biomass could harvest with two cuttings compared to single cut for fodder. These results are in conformity with the findings of Waseem and Badrul (1999).

The green fodder and dry matter yield was significantly higher with application nitrogen 
at $150 \mathrm{~N} \mathrm{ha}^{-1}\left(316.91 \mathrm{q}\right.$ and $74.95 \mathrm{q} \mathrm{ha}^{-1}$ respectively) as compared to nitrogen at 100 $\mathrm{kg} \mathrm{ha}{ }^{-1}\left(289.89 \mathrm{q}\right.$ and $58.64 \mathrm{q} \mathrm{ha}^{-1}$ respectively) (Table 1). This may be mainly because the nitrogen improved growth parameters viz., plant height, number of tillers $\mathrm{m}^{-1}$ row length, leaf: stem ratio. The nitrogen is directly involved in cell division, elongation, formation of nucleotides and coenzymes which resulted in increased meristematic activity and nitrogen is integral part of chlorophyll which plays important role in photosynthetic activity of leaves finally helped to accumulate more biomass. These results are in conformity with the findings of Dudhat et al., (2004), Sharma and Verma (2005), Sheoron and Rana (2006), Chotiya and Singh (2005), Singh et al., (2013) and Chouhan et al., (2015).

The interaction of varieties and cutting management were found significant in green fodder and dry matter yield. BAIF Bajra-1 with two cuts for green fodder followed by harvest for grain purpose recorded significantly higher green fodder and dry matter yield (393.62 q ha ${ }^{-1}$ and $104.75 \mathrm{q} \mathrm{ha}^{-1}$ ) (Table 1). This was mainly due to genetic potential of BAIF Bajra-1 made to absorb and assimilate more nitrogen which finally resulted in higher green fodder and dry matter yield. The results are in line with the findings of (Joon et al., 1993), and (Leva et al., 2004).

The interaction effect of variety BAIF Bajra-1 with application of nitrogen $150 \mathrm{~kg} \mathrm{ha}^{-1}$ found significant in dry matter yield (108.52 $\left.\mathrm{q} \mathrm{ha}^{-1}\right)$. The higher level of nitrogen increased the availability and uptake which resulted in higher plant height, number of tillers and leaf: stem ratio and led to higher photosynthesis which caused more dry matter production. Similar results were reported by Ramesh Babu et al., (1995), Tiwana et al., (2003), Sheoron et al., (2008) and (Phatan et al., 2007).
The interaction effect of two cuts for green fodder followed by harvest for grain purpose with application of nitrogen $150 \mathrm{~kg} \mathrm{ha}^{-1}$ recorded significantly higher dry matter yield (797.4 $\mathrm{q} \mathrm{ha}^{-1}$ ) compared to other combinations (Table 1). This was mainly due to more number of tiller and leaf: stem ratio contributed for higher dry matter yield.

Variety BAIF Bajra-1 with two cuts for green fodder followed by harvest for grain purpose along with application of nitrogen $150 \mathrm{~kg} \mathrm{ha}^{-1}$ $\left(135.28 \mathrm{q} \mathrm{ha}^{-1}\right)$ resulted in significantly higher dry matter yield (Table 1). This is mainly due to genetic make-up of the variety BAIF Bajra ${ }^{1}$ has made to uptake and utilizes the nitrogen more efficiently in enhancing all growth parameters like plant height, number of tillers and leaf: stem ratio which leads to higher green fodder yield and in turn increases dry matter accumulation and yield.

\section{Nutrient uptake by the crop $\left(\mathrm{kg} \mathrm{ha}^{-1}\right)$}

BAIF Bajra-1 variety recorded significantly higher nitrogen, phosphorus and potassium uptake $\left(174.26,17.85,142.75 \mathrm{~kg} \mathrm{ha}{ }^{-1}\right.$ respectively) as compared to variety GFB-1 (116.99, $12.51,108.86 \mathrm{~kg} \mathrm{ha}^{-1}$ respectively) and AVKB-19 (88.18, 9.69, 84.39 kg ha respectively) (Table 2 and Fig. 1). Single cut for green fodder followed by harvest for grain purpose has recorded significantly higher nitrogen, phosphorus and potassium uptake $\left(138.83,14.45,117.32 \mathrm{~kg} \mathrm{ha}^{-1}\right.$ respectively) as compared to the two cuts for green fodder followed by harvest for grain purpose (114.12, $12.26, \quad 106.63 \quad \mathrm{~kg} \mathrm{ha}^{-1}$ respectively).Application of nitrogen $150 \mathrm{~kg}$ $\mathrm{ha}^{-1}$ recorded significantly higher nitrogen, phosphorus and potassium uptake (146.33, $15.51,126.54 \mathrm{~kg} \mathrm{ha}^{-1}$ respectively) compared to nitrogen $100 \mathrm{~kg} \mathrm{ha}^{-1}(106.62,11.20,97.41$ $\mathrm{kg} \mathrm{ha}{ }^{-1}$ respectively). The interaction of varieties and cutting management were found significant. 
Table.1 Green forage yield (GFY) and Dry matter yield (DMY) of dual purpose pearl millet varieties as influenced by cutting and nitrogen management

\begin{tabular}{|c|c|c|c|c|c|}
\hline $\begin{array}{c}\text { Treatment } \\
\text { s }\end{array}$ & $\underset{1}{\operatorname{GFY}}\left(\mathrm{q} \mathrm{ha}^{-}\right.$ & $\underset{1}{\text { DMY }_{\text {(q }}}$ & Treatments & GFY (q ha $\left.{ }^{-1}\right)$ & DMY (q ha $_{1}$ \\
\hline \multicolumn{3}{|c|}{ Varieties $(\mathbf{V})$} & \multicolumn{3}{|c|}{ Interaction $(\mathbf{C} \times \mathbf{N})$} \\
\hline $\mathbf{V}_{1}$ & 365.21 & 92.47 & $\mathrm{C}_{1} \times \mathrm{N}_{1}$ & 263.28 & 53.22 \\
\hline $\mathbf{V}_{2}$ & 249.78 & 43.00 & $\mathrm{C}_{1} \times \mathrm{N}_{2}$ & 281.70 & 61.30 \\
\hline $\mathbf{V}_{3}$ & 295.21 & 64.90 & $\mathrm{C}_{2} \times \mathrm{N}_{1}$ & 316.50 & 64.06 \\
\hline S.Em.土 & 6.70 & 2.41 & $\mathrm{C}_{2} \times \mathrm{N}_{2}$ & 352.12 & 88.60 \\
\hline CD@ $5 \%$ & 19.65 & 7.07 & S.Em.土 & 7.74 & 2.79 \\
\hline \multicolumn{3}{|c|}{ Cutting management $(\mathrm{C})$} & CD@ $9 \%$ & NS & 8.17 \\
\hline $\mathbf{C}_{1}$ & 272.49 & 57.26 & \multicolumn{3}{|c|}{ Interaction $(\mathrm{V} \times \mathrm{C} \times \mathrm{N})$} \\
\hline $\mathbf{C}_{2}$ & 334.31 & 76.33 & $\mathrm{~V}_{1} \times \mathrm{C}_{1} \times \mathrm{N}_{1}$ & 335.53 & 78.65 \\
\hline S.Em.土 & 5.47 & 1.97 & $\mathrm{~V}_{1} \times \mathrm{C}_{1} \times \mathrm{N}_{2}$ & 338.08 & 81.75 \\
\hline CD@ $9 \%$ & 16.05 & 5.78 & $\mathrm{~V}_{1} \times \mathrm{C}_{2} \times \mathrm{N}_{1}$ & 360.00 & 74.21 \\
\hline \multicolumn{3}{|c|}{ Nitrogen level $(\mathbf{N})$} & $\mathrm{V}_{1} \times \mathrm{C}_{2} \times \mathrm{N}_{2}$ & 427.25 & 135.28 \\
\hline $\mathbf{N}_{1}$ & 289.89 & 58.64 & $\mathrm{~V}_{2} \times \mathrm{C}_{1} \times \mathrm{N}_{1}$ & 227.10 & 36.28 \\
\hline $\mathbf{N}_{2}$ & 316.91 & 74.95 & $\mathbf{V}_{2} \times \mathrm{C}_{1} \times \mathrm{N}_{2}$ & 245.28 & 43.39 \\
\hline S.Em. \pm & 5.47 & 1.97 & $\mathrm{~V}_{2} \times \mathrm{C}_{2} \times \mathrm{N}_{1}$ & 253.70 & 43.48 \\
\hline CD@ $9 \%$ & 16.05 & 5.78 & $\mathrm{~V}_{2} \times \mathrm{C}_{2} \times \mathrm{N}_{2}$ & 273.03 & 48.86 \\
\hline \multicolumn{3}{|c|}{ Interaction $(\mathrm{V} \times \mathrm{C})$} & $\mathrm{V}_{3} \times \mathrm{C}_{1} \times \mathrm{N}_{1}$ & 227.20 & 44.72 \\
\hline $\mathbf{V}_{1} \times C_{1}$ & 336.80 & 80.20 & $\mathbf{V}_{3} \times \mathrm{C}_{1} \times \mathrm{N}_{2}$ & 261.74 & 58.76 \\
\hline $\mathbf{V}_{1} \times C_{2}$ & 393.62 & 104.75 & $\mathrm{~V}_{3} \times \mathrm{C}_{2} \times \mathrm{N}_{1}$ & 335.81 & 74.48 \\
\hline $\mathrm{V}_{2} \times \mathrm{C}_{1}$ & 236.18 & 39.83 & $\mathbf{V}_{3} \times \mathrm{C}_{2} \times \mathrm{N}_{2}$ & 356.09 & 81.65 \\
\hline $\mathrm{V}_{2} \times \mathrm{C}_{2}$ & 263.37 & 46.17 & S.Em.土 & 13.40 & 4.82 \\
\hline $\mathrm{V}_{3} \times \mathrm{C}_{1}$ & 244.47 & 51.73 & CD@ $15 \%$ & $\mathrm{NS}$ & 14.15 \\
\hline $\mathrm{V}_{3} \times \mathrm{C}_{2}$ & 345.95 & 78.07 & CV (\%) & 9.16 & 9.72 \\
\hline S.Em.土 & 9.48 & 3.41 & \multirow{11}{*}{\multicolumn{3}{|c|}{$\begin{array}{l}\mathbf{V}_{1}: \text { BAIF Bajra-1 } \\
\mathbf{V}_{\mathbf{2}}: \text { AVKB-19 } \\
\mathbf{V}_{3}: \text { GFB-1 } \\
\mathbf{C}_{\mathbf{1}}: \text { Single cut at } 45 \text { days after sowing for } \\
\text { green fodder followed by harvest for grain } \\
\text { purpose. } \\
\mathbf{C}_{2}: \text { Two cuts ( } 1^{\text {st }} \text { at } 45 \text { days after sowing } \\
\left.\text { and } 2^{\text {nd }} \text { at } 40 \text { days after } 1^{\text {st }} \text { cut }\right) \text { for green } \\
\text { fodder followed by harvest for grain } \\
\text { purpose. }\end{array}$}} \\
\hline CD @ 5\% & 27.79 & 10.00 & & & \\
\hline \multicolumn{3}{|c|}{ Interaction $(\mathrm{V} \times \mathrm{N})$} & & & \\
\hline $\mathbf{V}_{1} \times \mathbf{N}_{1}$ & 347.77 & 76.43 & & & \\
\hline $\mathrm{V}_{1} \times \mathrm{N}_{2}$ & 382.67 & 108.52 & & & \\
\hline $\mathrm{V}_{2} \times \mathrm{N}_{1}$ & 240.40 & 39.88 & & & \\
\hline $\mathbf{V}_{2} \times \mathbf{N}_{2}$ & 259.15 & 46.13 & & & \\
\hline $\mathrm{V}_{3} \times \mathrm{N}_{1}$ & 281.50 & 59.60 & & & \\
\hline $\mathrm{V}_{3} \times \mathrm{N}_{2}$ & 308.92 & 70.20 & & & \\
\hline S.Em. \pm & 9.48 & 3.41 & & & \\
\hline CD@ $5 \%$ & NS & 10.00 & & & \\
\hline
\end{tabular}


Table.2 Nutrient uptake (Nitrogen, Phosphorus and potassium) of dual purpose pearl millet varieties as influenced by cutting and nitrogen management

\begin{tabular}{|c|c|c|c|c|c|c|c|}
\hline Treatments & $\begin{array}{c}\text { Nitroge } \\
\text { n }(\mathbf{k g} \\
\left.\mathbf{h a}^{-1}\right)\end{array}$ & $\begin{array}{c}\mathrm{P}_{2} \mathrm{O}_{5}(\mathrm{~kg} \\
\left.\mathrm{ha}^{-1}\right)\end{array}$ & $\begin{array}{c}\mathrm{K}_{2} \mathrm{O} \\
\left(\mathrm{kg} \mathrm{ha}^{-1}\right)\end{array}$ & Treatments & $\begin{array}{c}\text { Nitrogen } \\
\left(\mathrm{kg} \mathrm{ha}^{-1}\right)\end{array}$ & $\begin{array}{c}\mathbf{P}_{2} \mathbf{O}_{5} \\
\left(\mathrm{~kg} \mathrm{ha}^{-1}\right)\end{array}$ & $\begin{array}{c}\mathrm{K}_{2} \mathrm{O} \\
\left(\mathrm{kg} \mathrm{ha}^{-1}\right)\end{array}$ \\
\hline \multicolumn{4}{|c|}{ Varieties (V) } & \multicolumn{4}{|c|}{ Interaction $(\mathrm{C} \times \mathrm{N})$} \\
\hline $\mathbf{V}_{1}$ & 174.26 & 17.85 & 142.75 & $\mathrm{C}_{1} \times \mathrm{N}_{1}$ & 117.37 & 11.85 & 103.16 \\
\hline $\mathbf{V}_{2}$ & 88.18 & 9.69 & 84.31 & $\mathrm{C}_{1} \times \mathrm{N}_{2}$ & 160.28 & 17.03 & 131.46 \\
\hline $\mathbf{V}_{3}$ & 116.99 & 12.51 & 108.86 & $\mathrm{C}_{2} \times \mathrm{N}_{1}$ & 95.86 & 10.53 & 91.64 \\
\hline S.Em. \pm & 2.87 & 0.38 & 3.33 & $\mathrm{C}_{2} \times \mathrm{N}_{2}$ & 132.37 & 13.97 & 121.60 \\
\hline CD@ 5\% & 8.40 & 1.11 & 9.78 & S.Em.土 & 3.31 & 0.44 & 3.85 \\
\hline \multicolumn{4}{|c|}{ Cutting management $(\mathrm{C})$} & CD@ $@ 5 \%$ & $\mathrm{NS}$ & $\mathrm{NS}$ & $\mathrm{NS}$ \\
\hline $\mathbf{C}_{1}$ & 138.83 & 14.45 & 117.32 & \multicolumn{4}{|c|}{ Interaction $(\mathrm{V} \times \mathrm{C} \times \mathrm{N})$} \\
\hline $\mathbf{C}_{2}$ & 114.12 & 12.26 & 106.63 & $\mathrm{~V}_{1} \times \mathrm{C}_{1} \times \mathrm{N}_{1}$ & 164.54 & 14.96 & 130.14 \\
\hline S.Em.土 & 2.34 & 0.31 & 2.72 & $\mathrm{~V}_{1} \times \mathrm{C}_{1} \times \mathrm{N}_{2}$ & 230.93 & 25.02 & 167.43 \\
\hline CD@ $5 \%$ & 6.86 & 0.90 & 7.98 & $\mathrm{~V}_{1} \times \mathrm{C}_{2} \times \mathrm{N}_{1}$ & 109.02 & 11.98 & 104.23 \\
\hline \multicolumn{4}{|c|}{ Nitrogen level (N) } & $\mathrm{V}_{1} \times \mathrm{C}_{2} \times \mathrm{N}_{2}$ & 192.54 & 19.45 & 169.20 \\
\hline $\mathrm{N}_{1}$ & 106.62 & 11.20 & 97.41 & $\mathrm{~V}_{2} \times \mathrm{C}_{1} \times \mathrm{N}_{1}$ & 81.19 & 8.92 & 77.62 \\
\hline $\mathbf{N}_{2}$ & 146.33 & 15.51 & 126.54 & $\mathrm{~V}_{2} \times \mathrm{C}_{1} \times \mathrm{N}_{2}$ & 110.95 & 12.19 & 106.07 \\
\hline S.Em.土 & 2.34 & 0.31 & 2.72 & $\mathrm{~V}_{2} \times \mathrm{C}_{2} \times \mathrm{N}_{1}$ & 73.42 & 8.07 & 70.19 \\
\hline CD@ $9 \%$ & 6.86 & 0.90 & 7.98 & $\mathrm{~V}_{2} \times \mathrm{C}_{2} \times \mathrm{N}_{2}$ & 87.17 & 9.58 & 83.34 \\
\hline \multicolumn{4}{|c|}{ Interaction $(\mathrm{V} \times \mathrm{C})$} & $\mathrm{V}_{3} \times \mathrm{C}_{1} \times \mathrm{N}_{1}$ & 106.41 & 11.69 & 101.73 \\
\hline $\mathrm{V}_{1} \times \mathrm{C}_{1}$ & 197.73 & 19.98 & 148.78 & $\mathrm{~V}_{3} \times \mathrm{C}_{1} \times \mathrm{N}_{2}$ & 138.97 & 13.90 & 120.90 \\
\hline $\mathbf{V}_{1} \times \mathbf{C}_{2}$ & 150.78 & 15.71 & 136.71 & $\mathrm{~V}_{3} \times \mathrm{C}_{2} \times \mathrm{N}_{1}$ & 105.15 & 11.55 & 100.53 \\
\hline $\mathrm{V}_{2} \times \mathrm{C}_{1}$ & 96.06 & 10.55 & 91.85 & $\mathrm{~V}_{3} \times \mathrm{C}_{2} \times \mathrm{N}_{2}$ & 117.43 & 12.91 & 112.27 \\
\hline $\mathrm{V}_{2} \times \mathrm{C}_{2}$ & 80.30 & 8.81 & 76.76 & S.Em.. & 5.73 & 0.75 & 6.67 \\
\hline $\mathrm{V}_{3} \times \mathrm{C}_{1}$ & 122.68 & 12.8 & 111.31 & CD@ $9 \%$ & $\mathrm{NS}$ & $\mathrm{NS}$ & $\mathrm{NS}$ \\
\hline $\mathrm{V}_{3} \times \mathrm{C}_{2}$ & 111.28 & 12.23 & 106.40 & CV (\%) & \begin{tabular}{|l|l}
6.99 \\
\end{tabular} & 7.14 & 7.37 \\
\hline S.Em.土 & 4.05 & 0.53 & 4.71 & \multirow{11}{*}{\multicolumn{4}{|c|}{$\begin{array}{l}\mathbf{V}_{\mathbf{1}}: \text { BAIF Bajra-1 } \\
\mathbf{V}_{\mathbf{2}}: \text { AVKB-19 } \\
\mathbf{V}_{\mathbf{3}}: \text { GFB-1 } \\
\mathbf{C}_{\mathbf{1}} \text { : Single cut at } 45 \text { days after sowing for } \\
\text { green fodder followed by harvest for grain } \\
\text { purpose. } \\
\mathbf{C}_{\mathbf{2}}: \text { Two cuts }\left(1^{\text {st }} \text { at } 45 \text { days after sowing }\right. \\
\text { and } 2^{\text {nd }} \text { at } 40 \text { days after } 1^{\text {st }} \text { cut) for green } \\
\text { fodder followed by harvest for grain } \\
\text { purpose. } \\
\mathbf{N}_{1}: 100 \mathrm{~kg} \text { Nitrogen ha }{ }^{-1} \\
\mathbf{N}_{2}: 150 \mathrm{~kg} \text { Nitrogen ha }{ }^{-1}\end{array}$}} \\
\hline CD@ 5\% & 11.88 & 1.57 & NS & & & & \\
\hline \multicolumn{4}{|c|}{ Interaction $(\mathrm{V} \times \mathbf{N})$} & & & & \\
\hline $\mathrm{V}_{1} \times \mathrm{N}_{1}$ & 136.78 & 13.46 & 117.18 & & & & \\
\hline $\mathrm{V}_{1} \times \mathrm{N}_{2}$ & 211.73 & 22.23 & 168.31 & & & & \\
\hline $\mathrm{V}_{2} \times \mathrm{N}_{1}$ & 77.30 & 8.50 & 73.90 & & & & \\
\hline $\mathrm{V}_{2} \times \mathrm{N}_{2}$ & 99.06 & 10.88 & 94.70 & & & & \\
\hline $\mathrm{V}_{3} \times \mathrm{N}_{1}$ & 105.78 & 11.61 & 101.13 & & & & \\
\hline $\mathbf{V}_{3} \times \mathbf{N}_{2}$ & 128.2 & 13.40 & 116.58 & & & & \\
\hline S.Em. \pm & 4.05 & 0.53 & 4.71 & & & & \\
\hline CD@ $9 \%$ & 11.88 & 1.57 & 13.83 & & & & \\
\hline
\end{tabular}


Table.3 Available Nitrogen, Phosphorus and Potassium content in soil $\left(\mathrm{kg} \mathrm{ha}^{-1}\right)$ after harvest of crop as influenced by cutting and nitrogen management

\begin{tabular}{|c|c|c|c|c|c|c|c|}
\hline Treatments & $\begin{array}{l}\text { Avail. N } \\
\left(\text { kg ha }^{-1}\right)\end{array}$ & $\begin{array}{c}\text { Avail. } \mathbf{P}_{2} \mathrm{O}_{5} \\
\left(\mathrm{~kg} \mathrm{ha}^{-1}\right)\end{array}$ & $\begin{array}{c}\text { Avail. } \mathrm{K}_{2} \mathrm{O} \\
\left(\mathrm{kg} \mathrm{ha}^{-1}\right)\end{array}$ & Treatments & $\begin{array}{l}\text { Avail. N } \\
\left(\text { kg ha }^{-1}\right)\end{array}$ & $\begin{array}{c}\text { Avail. } \mathbf{P}_{2} \mathbf{O}_{5} \\
\left(\mathrm{~kg} \mathrm{ha}^{-1}\right)\end{array}$ & $\begin{array}{c}\text { Avail. } \mathrm{K}_{2} \mathrm{O} \\
\left(\mathrm{kg} \mathrm{ha}^{-1}\right)\end{array}$ \\
\hline \multicolumn{4}{|c|}{ Varieties $(\mathbf{V})$} & \multicolumn{4}{|c|}{ Interaction $(\mathrm{C} \times \mathrm{N})$} \\
\hline $\mathbf{V}_{1}$ & 167.57 & 22.75 & 114.21 & $\mathrm{C}_{1} \times \mathrm{N}_{1}$ & 162.70 & 21.54 & 113.44 \\
\hline $\mathbf{V}_{2}$ & 169.23 & 23.51 & 118.46 & $\mathrm{C}_{1} \times \mathrm{N}_{2}$ & 164.93 & 22.16 & 110.90 \\
\hline $\mathbf{V}_{3}$ & 172.08 & 22.88 & 116.47 & $\mathrm{C}_{2} \times \mathrm{N}_{1}$ & 174.71 & 24.63 & 119.78 \\
\hline S.Em.土 & 0.75 & 0.26 & 1.20 & $\mathrm{C}_{2} \times \mathrm{N}_{2}$ & 176.17 & 23.86 & 121.40 \\
\hline CD@ $5 \%$ & 2.21 & NS & $\mathrm{NS}$ & S.Em.土 & 0.87 & 0.30 & 1.39 \\
\hline \multicolumn{4}{|c|}{ Cutting management $(\mathrm{C})$} & CD @ 5\% & NS & 0.88 & NS \\
\hline $\mathrm{C}_{1}$ & 163.81 & 21.85 & 112.17 & \multicolumn{4}{|c|}{ Interaction $(\mathrm{V} \times \mathrm{C} \times \mathrm{N})$} \\
\hline $\mathbf{C}_{2}$ & 175.44 & 24.25 & 120.59 & $\mathrm{~V}_{1} \times \mathrm{C}_{1} \times \mathrm{N}_{1}$ & 162.30 & 21.95 & 111.13 \\
\hline S.Em. \pm & 0.61 & 0.21 & 0.98 & $\mathrm{~V}_{1} \times \mathrm{C}_{1} \times \mathrm{N}_{2}$ & 163.56 & 21.56 & 106.05 \\
\hline CD@ $5 \%$ & 1.81 & 0.63 & 2.88 & $\mathrm{~V}_{1} \times \mathrm{C}_{2} \times \mathrm{N}_{1}$ & 170.66 & 24.33 & 118.74 \\
\hline \multicolumn{4}{|c|}{ Nitrogen level $(\mathbf{N})$} & $\mathrm{V}_{1} \times \mathrm{C}_{2} \times \mathrm{N}_{2}$ & 173.75 & 23.61 & 120.90 \\
\hline $\mathbf{N}_{1}$ & 168.70 & 23.01 & 116.61 & $\mathrm{~V}_{2} \times \mathrm{C}_{1} \times \mathrm{N}_{1}$ & 160.33 & 21.37 & 117.22 \\
\hline $\mathbf{N}_{2}$ & 170.55 & 23.09 & 116.15 & $\mathrm{~V}_{2} \times \mathrm{C}_{1} \times \mathrm{N}_{2}$ & 163.05 & 23.01 & 111.62 \\
\hline S.Em. \pm & 0.61 & 0.21 & 0.98 & $\mathrm{~V}_{2} \times \mathrm{C}_{2} \times \mathrm{N}_{1}$ & 175.01 & 25.36 & 122.30 \\
\hline CD @ 5\% & 1.81 & NS & NS & $\mathrm{V}_{2} \times \mathrm{C}_{2} \times \mathrm{N}_{2}$ & 178.54 & 24.31 & 122.72 \\
\hline \multicolumn{4}{|c|}{ Interaction $(\mathbf{V} \times \mathbf{C})$} & $\mathrm{V}_{3} \times \mathrm{C}_{1} \times \mathrm{N}_{1}$ & 165.46 & 21.31 & 111.98 \\
\hline $\mathrm{V}_{1} \times \mathrm{C}_{1}$ & 162.93 & 21.76 & 108.59 & $\mathrm{~V}_{3} \times \mathrm{C}_{1} \times \mathrm{N}_{2}$ & 168.18 & 21.91 & 115.04 \\
\hline $\mathrm{V}_{1} \times \mathrm{C}_{2}$ & 172.20 & 23.74 & 119.82 & $\mathrm{~V}_{3} \times \mathrm{C}_{2} \times \mathrm{N}_{1}$ & 178.45 & 24.21 & 118.30 \\
\hline $\mathrm{V}_{2} \times \mathrm{C}_{1}$ & 163.68 & 22.19 & 114.42 & $\mathrm{~V}_{3} \times \mathrm{C}_{2} \times \mathrm{N}_{2}$ & 176.22 & 24.10 & 120.57 \\
\hline $\mathrm{V}_{2} \times \mathrm{C}_{2}$ & 176.78 & 24.84 & 122.51 & S.Em.土 & 1.50 & 0.52 & 2.40 \\
\hline $\mathrm{V}_{3} \times \mathrm{C}_{1}$ & 166.82 & 21.61 & 113.51 & CD @ 5\% & NS & NS & NS \\
\hline $\mathrm{V}_{3} \times \mathrm{C}_{2}$ & 177.33 & 24.16 & 119.44 & CV $(\%)$ & 6.08 & 8.93 & 7.68 \\
\hline S.Em. \pm & 1.06 & 0.36 & 1.70 & \multirow{11}{*}{\multicolumn{4}{|c|}{$\begin{array}{l}\mathbf{V}_{1}: \text { BAIF Bajra-1 } \\
\mathbf{V}_{2}: \text { AVKB-19 } \\
\mathbf{V}_{3} \text { : GFB-1 } \\
\mathbf{C}_{1} \text { : Single cut at } 45 \text { days after sowing for green fodder followed } \\
\text { by harvest for grain purpose. } \\
\mathbf{C}_{2} \text { : Two cuts }\left(1^{\text {st }} \text { at } 45 \text { days after sowing and } 2^{\text {nd }} \text { at } 40 \text { days }\right. \\
\text { after } 1^{\text {st }} \text { cut) for green fodder followed by harvest for grain } \\
\text { purpose. }\end{array}$}} \\
\hline CD@ $5 \%$ & 3.13 & NS & NS & & & & \\
\hline \multicolumn{4}{|c|}{ Interaction $(\mathbf{V} \times \mathbf{N})$} & & & & \\
\hline $\mathrm{V}_{1} \times \mathrm{N}_{1}$ & 166.48 & 23.14 & 114.94 & & & & \\
\hline $\mathrm{V}_{1} \times \mathrm{N}_{2}$ & 168.65 & 22.36 & 113.48 & & & & \\
\hline $\mathbf{V}_{2} \times N_{1}$ & 167.67 & 23.37 & 119.76 & & & & \\
\hline $\mathrm{V}_{2} \times \mathrm{N}_{2}$ & 170.80 & 23.66 & 117.17 & & & & \\
\hline $\mathrm{V}_{3} \times \mathrm{N}_{1}$ & 171.95 & 22.76 & 115.14 & & & & \\
\hline $\mathbf{V}_{3} \times \mathbf{N}_{2}$ & 172.20 & 23.01 & 117.80 & & & & \\
\hline S.Em.土 & 1.06 & 0.36 & 1.70 & & & & \\
\hline CD@ $5 \%$ & NS & NS & NS & & & & \\
\hline
\end{tabular}

Table.4 Economics of dual purpose pearl millet varieties as influenced by cutting and nitrogen management

\begin{tabular}{|c|c|c|c|c|}
\hline Treatments & $\begin{array}{c}\text { Total cost of } \\
\text { cultivation }\left(\mathrm{ha}^{-1}\right) \\
\end{array}$ & $\begin{array}{c}\text { Gross returns } \\
\left(\text { ha }^{-1}\right)\end{array}$ & $\begin{array}{c}\text { Net returns } \\
\left(\mathrm{ha}^{-1}\right)\end{array}$ & B:C ratio \\
\hline $\mathrm{V}_{1} \times \mathrm{C}_{1} \times \mathrm{N}_{1}$ & 16523 & 50404 & 33881 & 3.05 \\
\hline$V_{1} \times C_{1} \times N_{2}$ & 17174 & 60102 & 42928 & 3.49 \\
\hline $\mathbf{V}_{1} \times \mathbf{C}_{2} \times \mathbf{N}_{1}$ & 19523 & 46830 & 27307 & 2.39 \\
\hline $\mathbf{V}_{1} \times C_{2} \times N_{2}$ & 20174 & 56785 & 36611 & 2.81 \\
\hline $\mathrm{V}_{2} \times \mathrm{C}_{1} \times \mathrm{N}_{1}$ & 16523 & 35286 & 18763 & 2.13 \\
\hline $\mathrm{V}_{2} \times \mathrm{C}_{1} \times \mathrm{N}_{2}$ & 17174 & 43187 & 26013 & 2.51 \\
\hline $\mathrm{V}_{2} \times \mathrm{C}_{2} \times \mathrm{N}_{1}$ & 19523 & 34205 & 14682 & 1.75 \\
\hline $\mathbf{V}_{2} \times C_{2} \times N_{2}$ & 20174 & 38454 & 18280 & 1.90 \\
\hline $\mathrm{V}_{3} \times \mathrm{C}_{1} \times \mathrm{N}_{1}$ & 16523 & 39875 & 23352 & 2.41 \\
\hline $\mathrm{V}_{3} \times \mathrm{C}_{1} \times \mathrm{N}_{2}$ & 17174 & 45229 & 28055 & 2.63 \\
\hline $\mathbf{V}_{3} \times C_{2} \times N_{1}$ & 19523 & 43330 & 23807 & 2.21 \\
\hline $\mathbf{V}_{3} \times \mathbf{C}_{2} \times \mathbf{N}_{2}$ & 20174 & 46859 & 26685 & 2.32 \\
\hline
\end{tabular}




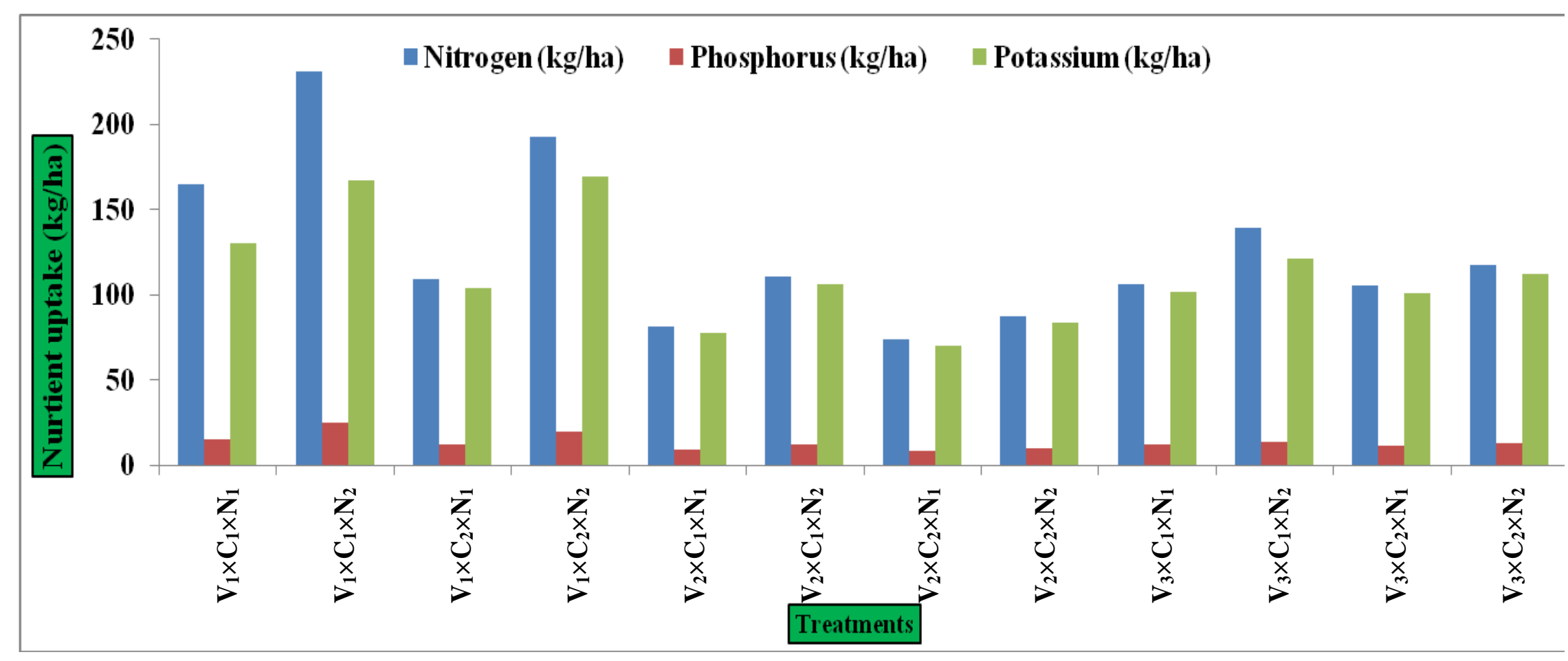

Fig. 1: Nutrient uptake $\left(\mathrm{kg} \mathrm{ha}^{-1}\right)$ of dual purpose pearl millet varieties as influenced by cutting and nitrogen management

\section{Varieties:}

$\mathbf{V}_{1}$ : BAIF Bajra-1

$\mathbf{V}_{2}$ : AVKB-19

V: GFB-1

\section{Cutting management:}

$\mathbf{C}_{1}$ : Single cut at 45 days after sowing for green fodder followed by harvest for grain purpose.

$\mathbf{C}_{2}$ : Two cuts ( $1^{\text {st }} 45$ days after sowing and

$2^{\text {nd }}$ at 40 days after $1^{\text {st }}$ cut) for green fodder

followed by harvest for grain purpose.
Nitrogen levels:

$\mathbf{N}_{1}: 100 \mathrm{~kg}$ Nitrogen /ha

$\mathbf{N}_{2}: 150 \mathrm{~kg}$ Nitrogen /ha 


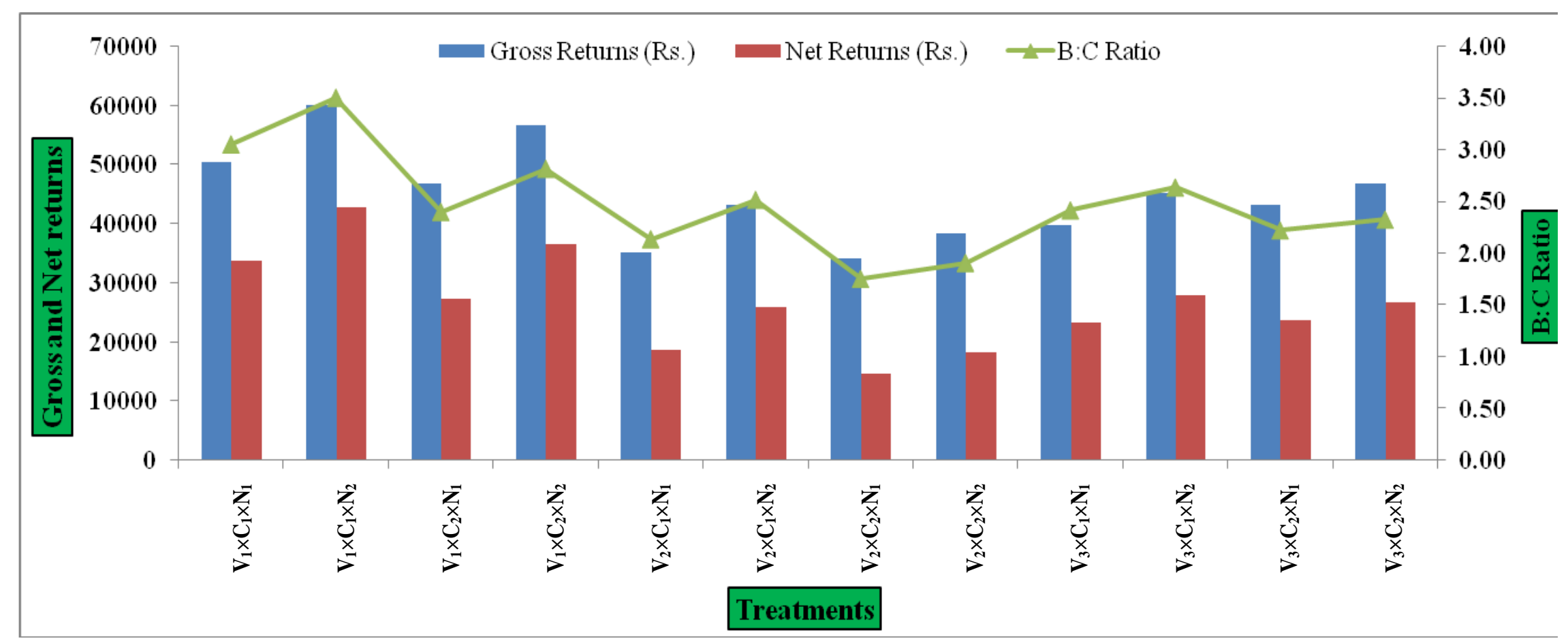

Fig. 2 : Economics of dual purpose pearl millet varieties as influenced by cutting and nitrogen management

\begin{tabular}{lll} 
Varieties: & \multicolumn{1}{c}{ Cutting management: } & Nitrogen levels: \\
V1: BAIF Bajra-1 & $\mathbf{C}_{1}$ : Single cut at 45 days after sowing for & $\mathbf{N}_{1}: 100 \mathrm{~kg}$ Nitrogen $/$ ha \\
$\mathbf{V}_{2}:$ AVKB-19 & green fodder followed by harvest for grain purpose. & $\mathbf{N}_{2}: 150 \mathrm{~kg}$ Nitrogen $/ \mathrm{ha}$ \\
$\mathbf{V}_{3}$ : GFB-1 & $\mathbf{C}_{2}:$ Two cuts $\left(1^{\text {st }} 45\right.$ days after sowing and & \\
& $2^{\text {nd }}$ at 40 days after $1^{\text {st }}$ cut $)$ for green fodder & \\
& followed by harvest for grain purpose. &
\end{tabular}


BAIF Bajra-1 with single cut for green fodder followed by harvest for grain purpose recorded significantly higher nitrogen and phosphorus uptake $\left(197.73,19.98 \mathrm{~kg} \mathrm{ha}^{-1}\right.$ respectively).BAIF Bajra-1 variety with application of nitrogen $150 \mathrm{~kg} \mathrm{ha}^{-1}$ found significant in NPK uptake $(211.73,22.23$, $168.31 \mathrm{~kg} \mathrm{ha}^{-1}$ respectively) (Table 2 and Fig. 1). The interaction of single cut for green fodder later leave it for grain purpose with nitrogen $150 \mathrm{~kg} \mathrm{ha}^{-1}$ found non-significant. The interaction of variety, cutting and nitrogen management were found nonsignificant. The higher nitrogen, phosphorus and potassium uptake $(230.93,25.02,167.43$ $\mathrm{kg} \mathrm{ha}{ }^{-1}$ ) was noticed in the variety BAIF Bajra-1 with single cut for green fodder followed by harvest for grain purpose along with application of nitrogen $150 \mathrm{~kg} \mathrm{ha}^{-1}$. The higher nutrient uptake was mainly due to the higher green fodder yield, dry matter yield, grain yield and stover yield. The results were conformity with the findings of Singh et al., (1983), Amrutkar et al., (1985), Yadav, (1988), Agarwal et al., (1992).

\section{Available soil nutrients ( $\left.\mathrm{kg} \mathrm{ha}^{-1}\right)$}

The available nutrient status in the soil differed significantly. The lower available NPK was observed in variety BAIF Bajra-1 $\left(167.57,22.75,114.21 \mathrm{~kg} \mathrm{ha}^{-1}\right.$ respectively) as compared to the variety GFB-1 (172.08, $22.28,116.47 \mathrm{~kg} \mathrm{ha}^{-1}$ respectively) and AVKB-19 (169.23, 23.51, $118.46 \mathrm{~kg} \mathrm{ha}^{-1}$ respectively). There was no significant difference between GFB-1 and AVKB-19 varieties. The single cut for green fodder followed by harvest for grain purpose has recorded significantly higher available nitrogen content (163.81 $\left.\mathrm{kg} \mathrm{ha}^{-1}\right)$, lower phosphorus and potassium content (21.85, $112.7 \mathrm{~kg} \mathrm{ha}^{-1}$ ) as compared to the two cuts for green fodder followed by harvest for grain purpose $\left(175.44,24.25,120.59 \mathrm{~kg} \mathrm{ha}{ }^{-1}\right.$ respectively). Application of $150 \mathrm{~kg}$ nitrogen ha $^{-1}$ recorded significantly higher available nitrogen and phosphorus content (170.55, $23.09 \mathrm{~kg} \mathrm{ha}^{-1}$ ) as compared to $100 \mathrm{~kg}$ nitrogen $\mathrm{ha}^{-1}\left(168.70,23.01 \mathrm{~kg} \mathrm{ha}^{-1}\right)$. The interaction of variety and cutting management were found significant with respect to nitrogen. BAIF Bajra-1 with single cut for green fodder followed by harvest for grain purpose recorded significantly lower available nitrogen content $\left(162.93 \mathrm{~kg} \mathrm{ha}^{-1}\right)$. The other interaction effects were found to be nonsignificant (Table 3).

\section{Economics}

Higher gross returns ( $\square 60102 \mathrm{ha}^{-1}$ ), net returns $\left(\square 42928 \mathrm{ha}^{-1}\right)$ and B:C ratio (3.49) were recorded in BAIF Bajra-1 with single cut for green fodder followed by harvest for grain purpose along with application nitrogen $150 \mathrm{~kg} \mathrm{ha}^{-1}$. Lower gross returns $(\square 34205$ $\left.\mathrm{ha}^{-1}\right)$, net returns $\left(\square 14682 \mathrm{ha}^{-1}\right)$ and B:C ratio (1.75) were obtained in AVKB-19 with two cuts for green fodder followed by harvest for grain purpose along with application of nitrogen $100 \mathrm{~kg} \mathrm{ha}^{-1}$ (Table 4 and Fig. 2). The higher $\mathrm{B}$ : $\mathrm{C}$ ratio in above interactions was mainly due to the higher grain and stover yield along with the green fodder increased gross returns.

The interaction of varieties and cutting management were found significant in green fodder and dry matter yield. BAIF Bajra-1 with two cuts for green fodder followed by harvest for grain purpose recorded significantly higher green fodder and dry matter yield (393.62 $\mathrm{q} \mathrm{ha}^{-1}$ and $104.75 \mathrm{q} \mathrm{ha}^{-1}$ ). The higher nitrogen, phosphorus and potassium uptake was noticed in the variety BAIF Bajra-1 with single cut for green fodder followed by harvest for grain purpose along with application of nitrogen $150 \mathrm{~kg} \mathrm{ha}^{-1}$. Higher B: C ratio was recorded with BAIF Bajra-1 with single cut for fodder followed by harvest for grain purpose along with 
application of nitrogen $150 \mathrm{~kg} \mathrm{ha}^{-1}$ (3.49). The next best combination was BAIF Bajra-1 with single cut for fodder purpose followed by harvest for grain purpose along with application of $100 \mathrm{~kg}$ of nitrogen ha $\mathrm{h}^{-1}(3.05)$. The higher $\mathrm{B}$ : $\mathrm{C}$ ratio in above interactions was mainly due to the higher grain and stover yield along with the green fodder increased gross returns.

\section{Acknowledgement}

The authors are thankful to the scheme head AICRP on Forage crops, Zonal Agricultural Research Station, V.C. Farm, Mandya for providing research facilities to carry out this experiment.

\section{References}

Agrawal, Patel, A. S., Sadhu, A. C. and Patel, M. P., 1992. Effect of Zinc, FYM and fertility levels on yield and quality of forage maize. Forage Research. 32(4): 209-212.

Amrutkar, V. R., Deshmukh, A. P. and Desale, J. S., 1985. Effect of nitrogen and phosphorus fertilization on the chemical composition and in vitro dry matter digestability of giant bajra. Journal of Maharashtra Agriculture University. 10(1): 93-94.

Anonymous, 2006. Hand book of Agriculture, ICAR, New Delhi. pp. 1353-1355.

Anonymous, 2009. Post harvest management of crop residues/grasses/fodder crops and their value addition for sustaining livestock. Winter School, Indian Grassland and Fodder Research Institute, Jhansi. pp. 13-23.

Bali, A. S., Shah, M. H., and Hasan, B. 1998. Influence of various nitrogen, phosphorus and seed rates on herbage yield and quality of newly released oat genotype SK-O-7 in temperate Kashmir. Forage Research. 24(2): 67-
70.

Chotiya, A., and Singh, P. 2005. Effect of graded levels of nitrogen and phosphorus on sorghum fodder production. Forage Research. 31(3): 218-219.

Chouhan, M., Gudadhe, N. N., Kumar, D., Kumawat, A. K. and Kumar, R. 2015. Transplanting dates and nitrogen levels influences on growth, yield attributes and yield of summer pearlmillet. The Bioscan. 10(3): 1295-1298

Dudhat, M. S., Savalia, M. G. and Ramdevputra, M. V. 2004.Response of forage maize to nitrogen and phosphorous levels. Forage Research. 30(1): 34-35.

Gomez, K. A. and Gomez, A. K., 1984. Statistical procedures for Agricultural Research, $2^{\text {nd }}$ Ed: John Wiley and Sons, New York, pp. 105-114.

Jackson, M. K., 1973. Soil chemical analysis. Prentice-Hall. Inc. Engle Wood Cliffs, New Jersey.

Jackson, M. L., 1967. Soil Chemical Analysis, Ed. Prentice Hall of India Pvt. Ltd., New Delhi, pp. 183-192.

Joon, R. K., Yadav, B. D., Rana, D. S. and Panwar, V. S. 1993.Response of forage sorghum to cutting management. Forage Research. 19(2): 233-236.

Leva, R. L., Patel, L. K., Mungra, K. D. and Pansuriya, A. G. 2004. Evaluation of promising forage sorghum genotypes for ratooning ability under varying fertility levels. Forage Research. 30(1): 45-48.

Panse, V. G. and Sukhatme, P. V. 1967. Statistical methods for agricultural workers. ICAR, Publ., New Delhi, p.359.

Phatan, S. H., Bhilare, R. L., Nawale, K. B. and Jadhav, V. T. 2007. Response of multicut oat varieties to nitrogen levels. Forage Research. 32(4): 269-270.

Ramesh Babu, Subhashgumaste, Patil, T. C. 
and Prabhakar, A. S. 1995.Effect of stage of cutting, nitrogen and phosphorous levels on forage pearl millet. Forage Research. 20(4): 225231.

Rana, D. S., Bhagatsingh and Joshi, U.N. 2009.Response of oat genotypes to nitrogen levels. Forage Research. 35(3): 184-185.

Reddy, A. A., Malik, D., Singh, I. P., Ardeshna, N. J., Kundu, K. K. Rao, P., Gupta S. K., Sharma, R. and Gajanan, 2012. Demand and supply for pearl millet Grain and fodder by 2020 in Western India. ASI. March, 2012. p. 635.

Sharma, K. C. and Verma, R. S. 2005. Effect of $\mathrm{N}$ and $\mathrm{P}$ and bio-fertilizers on the yield, marginal rate of return, energy relationship, economics and residual effect on soil in fodder oats (Avena sativa L.). Forage Research. 31(2): 118 112.

Sheoron, R. S. and Rana, D. S. 2006. Relative efficiency of azotobacter and nitrogen fertilizer in forage sorghum (Sorghum bicolor L.) under semi-arid conditions. Forage Research. 32(2): 65-68.

Sheoron, R. S., Yadav, N. S., Tiwana, U. S. and Joshi, U. N. 2008. Multi-locational evaluation of promising oat (Avena sativa L.) varieties for forage yield and quality under varying nitrogen levels. Forage Research. 34(2): 90-93.
Singh, G. D., Keshwa, G. L. and Khurana, G. P. 1983. Effect of doses and application of nitrogen on quality and quantity of bajra fodder. Trans. Isdt. 9(1): 8-11.

Singh, P., Rana, N. S., Shukla, U. N, Kumar, S. S. K. and Kumar, K. 2013. Effect of genotypes and nitrogen levels on production potential of maize (Zea mays L.) under Indo-Gangatic plane zone of Western U.P. The Bioscasn. 8(3): 777781.

Subbiah, B. V. and Asija, G. L., 1956, A rapid procedure for the determination of available nitrogen in soils. Current Science.31: 196.

Tiwana, U. S., Puri, K. P. and Sukhpreetsingh, 2003. Fodder yield and quality of multicut pearl millet (Pennisetum glaucum L.) as influenced by nitrogen and phosphorus under Punjab conditions. Forage Research. 28(4): 190-193.

Waseem, A. S. and Badrul, H. 1999. Grain and fodder yield of oats (Avena sativa L.) as influenced by nitrogen levels and cutting schedules. Forage Research. 24(4): 185-190.

Yadav, J. P. 1988. Effect of different form of nitrogen fertilization and levels of zinc on growth, yield and quality of pearl millet. M.Sc. (Agri.) Thesis, submitted to Rajasthan Agriculture University Bikaner.

\section{How to cite this article:}

Manjanagouda S. Sannagoudar, B.S. Lalitha, G.K. Prajwal Kumar, Prashant and Bhavya, V. 2017. Effect of Varieties, Cutting and Nitrogen Management on Green Fodder Yield, Nutrient Uptake, Available Soil Nutrient Status and Economics of Dual Purpose Pearl Millet (Pennisetum glaucum L.). Int.J.Curr.Microbiol.App.Sci. 6(12): 214-226. doi: https://doi.org/10.20546/ijcmas.2017.612.027 